\title{
Non-Hermitian Bulk-Boundary Correspondence in Periodically Driven System
}

\author{
Yang Cao, ${ }^{1}$ Yang $\mathrm{Li}^{1}{ }^{1}$ and Xiaosen Yang $^{1, *}$ \\ ${ }^{1}$ Department of physics, Jiangsu University, Zhenjiang, 212013, China
}

(Dated: August 4, 2020)

\begin{abstract}
Bulk-boundary correspondence, connecting the bulk topology and the edge states, is an essential principle of the topological phases. However, the bulk-boundary correspondence is broken down in general non-Hermitian systems. In this paper, we construct one-dimensional non-Hermitian Su-Schrieffer-Heeger model with periodic driving that exhibits non-Hermitian skin effect: all the eigenstates are localized at the boundary of the systems, whether the bulk states or the zero and the $\pi$ modes. To capture the topological properties, the non-Bloch winding numbers are defined by the non-Bloch periodized evolution operators based on the generalized Brillouin zone. Furthermore, the non-Hermitian bulk-boundary correspondence is established: the non-Bloch winding numbers $\left(W_{0, \pi}\right)$ characterize the edge states with quasienergies $\epsilon=0, \pi$. In our non-Hermitian system, a novel phenomenon can emerge that the robust edge states can appear even when the Floquet bands are topological trivial with zero non-Bloch band invariant, which is defined in terms of the non-Bloch effective Hamiltonian. We also show that the relation between the non-Bloch winding numbers $\left(W_{0, \pi}\right)$ and the non-Bloch band invariant $(\mathcal{W}): \mathcal{W}=W_{0}-W_{\pi}$.
\end{abstract}

Topological materials have robust topological edge states and can be characterized by the bulk topological invariants which is defined in terms of the Bloch Hamiltonian on Brillouin zone(BZ) [1-7]. The topological surface states are connected with the bulk topological classification by the bulkboundary correspondence[5-8], an essential principle of the topological phases.

Recently, non-Hermitian systems, described by the nonHermitian Hamiltonian, have been attracting much attention in many fields of physics [9-63]. In particular, the conventional bulk-boundary correspondence is broken in general non-Hermitian systems, which exhibits the non-Hermitian skin effect [23, 24, 64-81]. The wave functions of the nonHermitian systems with open boundary condition(OBC) do not extend over the bulk but are localized at the boundaries, contrast to the Hermitian systems. To depict the topological properties of the non-Hermitian systems, the BZ should be extended into the generalized Brillouin zone(GBZ) in complex plane [23-25, 82-90]. The deviation between the GBZ and the BZ results in the non-Hermitian skin effect and the breakdown of the Bloch bulk-boundary correspondence[23$26,82-86,91]$. The universal topological invariants are defined by the non-Bloch Hamiltonian based on GBZ. The nonBloch topological invariants capture the topological properties of the non-Hermitian systems like the robust topological edge modes[23, 24, 83], the unidirectional edge motion[24, 88, 92] and so on. Thus, the non-Hermitian bulk-boundary correspondence is well defined for general non-Hermitian systems and has been experimentally observed in photons[25] and metamaterials [55, 93, 94].

In addition, periodic driving offers a new fruitful platform for creating topological phenomena with high tunability, even to create the fundamentally new topological states without static counterparts[95-109]. For instance, the robust topological edge modes can appear at the boundary of the systems even though all the band invariants are zero. Due to the periodicity in time, there exist two types of robust topological edge

\footnotetext{
*yangxs@ujs.edu.cn
}

modes: zero modes and $\pi$ modes, which also has no static counterpart[97, 98].

In this paper, we interplay the non-Hermiticity and the periodic driving in one-dimensional Su-Schrieffer-Heeger(SSH) model by constructing the non-Bloch topological invariants in GBZ [23, 25, 91, 110]. First, the frequency-domain quasienergy spectra are given both in the OBC and the periodic boundary condition( $\mathrm{PBC})$. There is a sharp difference between the spectra in the two conditions. We find that the periodic driving induces two types of topological edge modes with quasienergies $\epsilon=0$ and $\epsilon=\pi$ in OBC. The robust edge modes cannot be predicted by the winding number defined on BZ. To capture the robust topological edge modes, we construct the non-Bloch winding numbers defined in terms of the non-Bloch periodized evolution operators based on GBZ. The non-Bloch winding numbers exactly predict the zero modes and the $\pi$ modes. Then, the non-Hermitian bulk-boundary correspondence is established for the non-Hermitian system with periodic driving. Secondly, we give the two equivalent definitions of non-Bloch band invariant defined in terms of the frequency-domain and the effective Hamiltonian on GBZ. We find that the robust edge modes can appear when the Floquet bands are topological trivial with zero non-Bloch band invariant. Lastly, we show that the deviation between the GBZ and BZ induces the non-Hermitian skin effect: all the eigenstates are localized at the boundaries of the system, whether the bulk states or the topological zero and $\pi$ modes. We also give the phase diagram of the system by the non-Bloch band theory and the spectra of Floquet Hamiltonian in OBC.

Non-Hermitian Bloch Hamiltonian.- We consider a nonHermitian SSH model[23] with periodic driving. The Bloch Hamiltonian can be written as following:

$$
H(k, t)=\left[d_{x}+\lambda \cos (\omega t)\right] \sigma_{x}+\left[d_{y}+i \gamma\right] \sigma_{y},
$$

where $d_{x}=t_{1}+t_{2} \cos (k), d_{y}=t_{2} \sin (k)$ and $\sigma_{x, y}$ are the Pauli matrices. $\gamma$ and $\lambda$ are the strengths of non-Hermitian and periodic driving respectively. The Hamiltonian has a chiral symmetry[3] $\sigma_{z} H(k, t) \sigma_{z}=-H(k,-t)$ and periodicity $H(k, t)=H(k, t+T)$ with periodic time $T=2 \pi / \omega$. The chiral symmetry ensures the quasienergies appear in $(E,-E)$ pairs. 

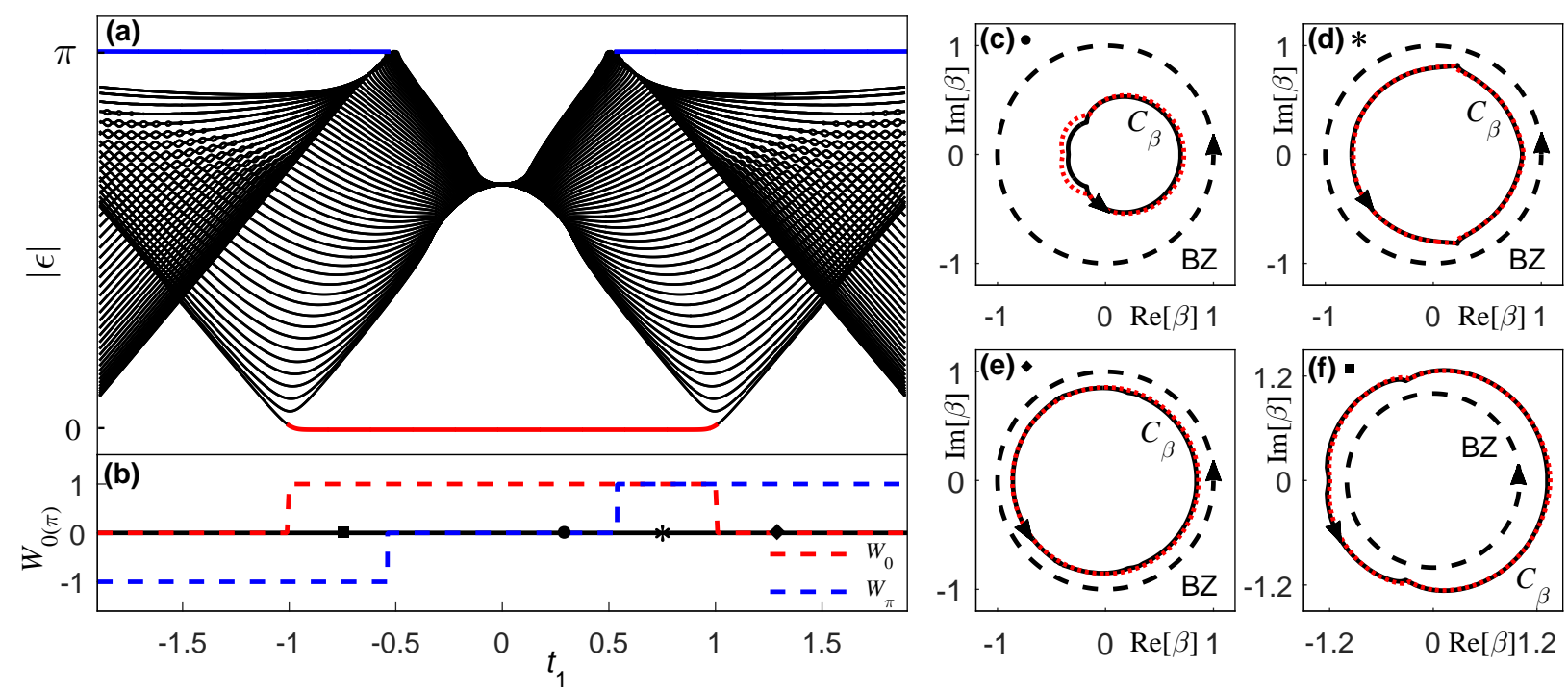

FIG. 1. Non-Hermitian Bulk-Boundary Correspondence for Edge States with quasienergies $\epsilon=0$, $\pi$ : (a) Quasienergy spectrum in open boundary condition and (b) non-Bloch winding numbers with the parameters: $t_{2}=1, \gamma=0.2, \lambda=0.5$ and $\omega=3$. (c)-(f) The generalized Brillouin zone of the four marked topological different phases in (b) determined by non-Bloch Floquet Hamiltonian (solid) and non-Bloch effective Hamiltonian (dotted). The values of the parameters are (c) $t_{1}=0.3$, (d) $t_{1}=0.75$, (e) $t_{1}=1.3$ and (f) $t_{1}=-0.75$. The Brillouin zone is a unit circle (dashed). The non-Bloch winding numbers $\left(W_{0, \pi}\right)$, defined on the generalized Brillouin zone, characterize the zero and $\pi$ modes in open boundary condition.

Non-Hermitian Bulk-Boundary Correspondence.- For simplicity, we numerical calculate the quasienergy spectra of the non-Hermitian Hamiltonian by the frequency-domain formulation[97, 98]. Let us start from the time-dependent Schrödinger equation for the Floquet state in band $n$ :

$$
i \partial_{t}\left|\psi_{n, R}(k, t)\right\rangle=H(k, t)\left|\psi_{n, R}(k, t)\right\rangle,
$$

and take the Floquet theorem and the Fourier transformation to the frequency domain, $\left|\psi_{n, R}(k, t)\right\rangle=$ $\exp \left[-i \varepsilon_{n}(k) t\right] \sum_{m} \exp (i m \omega t)\left|\psi_{n, R}^{m}(k)\right\rangle$, where $\quad \varepsilon_{n}$ is the quasienergy and $\left|\psi_{n, R}^{m}(k)\right\rangle$ is right wave vector. In frequency domain framework, the Schrödinger equation becomes

$$
\sum_{m^{\prime}} \mathcal{H}_{m, m^{\prime}}(k)\left|\psi_{n, R}^{m^{\prime}}(k)\right\rangle=\varepsilon_{n}(k)\left|\psi_{n, R}^{m}(k)\right\rangle,
$$

with the Floquet Hamiltonian $\mathcal{H}_{m, m^{\prime}}(k)=m \omega \delta_{m, m^{\prime}} \mathbf{I}+H_{m-m^{\prime}}(k)$ and $H_{m}(k)=\frac{1}{T} \int_{0}^{T} d t H(k, t) \exp (-i m \omega t)$. To be more explicit, the Floquet Hamiltonian reads

$$
\mathcal{H}=\left(\begin{array}{ccccc}
\cdots & & & & \\
& H_{0}+\omega & H_{1} & 0 & \\
& H_{-1} & H_{0} & H_{1} & \\
& 0 & H_{-1} & H_{0}-\omega & \\
& & & & \ldots
\end{array}\right),
$$

here, $H_{0}=d_{x} \sigma_{x}+\left[d_{y}+i \gamma\right] \sigma_{y}$ and $H_{ \pm 1}=\frac{\lambda}{2} \sigma_{x}$. The eigenvalues of the Floquet Hamiltonian are the quasienergies of the nonHermitian system. The quasienergies are paired with $(E,-E)$ for the chiral symmetry $C^{-1} \mathcal{H C}=-\mathcal{H}[102]$.

To show the quasienergy of the Floquet Hamiltonian $\mathcal{H}(k)$, we need to make a truncation on the Floquet Hamiltonian for its infinite rank. Fig.1(a) shows the dimensionless quasienergy $\epsilon=\varepsilon T$ of Floquet Hamiltonian as functions of $t_{1}$ in OBC with $t_{2}=1, \gamma=0.2, \lambda=0.5$ and $\omega=3$. In the presence of periodic driving, unconventional gap can emerge around quasienergy $\epsilon=\pi$, which enables the stability of robust nontrivial $\pi$ modes. Thus, two topological different types of the nontrivial edge modes can exist at the two separated gaps with quasienergies $\epsilon=0, \pi$. This phenomena seeming the same as the cases of Hermitian systems, while they are utterly different in topological characterizing and non-Hermitian skin effect. In the non-Hermitian systems, the two types of robust edge modes can be characterized by the topological invariants not rely on the Bloch band theory like the Hermitian cases, but on the non-Bloch band theory in generally. Fig.1(b) shows the non-Bloch winding numbers $W_{0, \pi}$ as functions of $t_{1}$, which are defined in terms of nonBloch periodized evolution operators based on GBZ $C_{\beta}$ and can exactly characterize the numbers of the two robust topological different edge modes.

Generalized Brillouin Zone and Non-Bloch Winding Numbers.- The precise description of the non-Hermitian systems relies on the non-Bloch band theory based on the GBZ. To determine the GBZ, we rewrite the Bloch Floquet Hamiltonian $\mathcal{H}(k)$ in terms of $\beta=e^{i k}$ as non-Bloch Floquet Hamiltonian $\mathcal{H}(\beta)$. Then, the eigenvalue equation $\operatorname{det}[\mathcal{H}(\beta)-E]=0$ is an algebraic equation for $\beta$ with even degree. Due to $2 \pi$ modules of the quasienergies $\epsilon$, we constrain the quasienergy in one period such as $\epsilon \in[-\pi, \pi]$. The solutions are numbered as $\beta_{i}(i=1,2, \ldots, 2 N)$ and satisfy $\left|\beta_{1}\right| \leq\left|\beta_{2}\right| \leq \ldots \leq\left|\beta_{2 N}\right|$. Thus, the GBZ $C_{\beta}$ can be determined by the trajectory of $\beta_{N}$ and $\beta_{N+1}$ with $\left|\beta_{N}\right|=\left|\beta_{N+1}\right|[23,25,83]$ as shown in Fig.1(c)-(f) for four marked points in Fig.1(b) with $t_{1}=0.3,0.75,1.3$ and -0.75 . GBZ, always deviates from $\mathrm{BZ}$ in general, is smaller than BZ for Fig.1(c)-(e) and larger than BZ for Fig.1(f). 
The non-Bloch time-evolution operator $U(\beta, t)$ can be got by rewriting the Bloch Hamiltonian $H(k, t)$ and is defined as

$$
U(\beta, t)=\mathcal{T} \exp \left[-i \int_{0}^{t} d t^{\prime} H\left(\beta, t^{\prime}\right)\right],
$$

where $\mathcal{T}$ is the time-ordering operator. The non-Bloch time-evolution operator satisfies the differential equation $i \partial_{t} U(\beta, t)=H(\beta, t) U(\beta, t)$. For one period, the non-Bloch Floquet operator $U(\beta, T)$ can be expanded as $U(\beta, T)=$ $\sum_{n} \lambda_{n}(\beta)\left|\psi_{n, R}(\beta)\right\rangle\left\langle\psi_{n, L}(\beta)\right|$. When there is a gap in the spectrum of the non-Bloch Floquet operator around $e^{-i \epsilon}$, we can define an unambiguous non-Bloch effective Hamiltonian as

$$
H_{\mathrm{eff}}^{\epsilon}(\beta)=\frac{i}{T} \ln _{-\epsilon} U(\beta, T) .
$$

The subscript $\epsilon$ has been introduced as the branch cut which plays an essential ingredient in the construction of topological invariants for non-Bloch Floquet systems. We take $\ln _{\epsilon} e^{i \phi}=i \phi$ for $\epsilon-2 \pi<\phi<\epsilon$. Then, the effective Hamiltonian breaks the chiral symmetry with $\sigma_{z} H_{\text {eff }}^{\epsilon}(\beta) \sigma_{z}=-H_{\text {eff }}^{-\epsilon}(\beta)+\omega$ and can be rewritten as $H_{\text {eff }}^{\epsilon}(\beta)=\frac{i}{T} \sum_{n} \ln _{-\epsilon}\left(\lambda_{n}(\beta)\right)\left|\psi_{n, R}(\beta)\right\rangle\left\langle\psi_{n, L}(\beta)\right|$. The effective Hamiltonian only captures the stroboscopic evolution at the quasienergy of $\epsilon$ and lost the information of timeevolution within each period. So, the non-Bloch topological invariants are defined by the non-Bloch periodized evolution operator, which can be written as following:

$$
U_{\epsilon}(\beta, t)=U(\beta, t) \exp \left[i H_{\mathrm{eff}}^{\epsilon}(\beta) t\right],
$$

with periodicity $U_{\epsilon}(\beta, t+T)=U_{\epsilon}(\beta, t)$. As for the chiral symmetry of the non-Bloch Hamiltonian, non-Bloch periodized evolution operator has the chiral symmetry only at half-period with quasienergy $\epsilon=0, \pi$ :

$$
\begin{aligned}
& \sigma_{z} U_{0}(\beta, T / 2) \sigma_{z}=-U_{0}(\beta, T / 2), \\
& \sigma_{z} U_{\pi}(\beta, T / 2) \sigma_{z}=+U_{\pi}(\beta, T / 2) .
\end{aligned}
$$

Therefore, $U_{0}(\beta, T / 2)$ and $U_{\pi}(\beta, T / 2)$ are off-diagonal and diagonal respectively, which can be written as following:

$$
\begin{aligned}
& U_{0}(\beta, T / 2)=\left(\begin{array}{cc}
0 & U_{0}^{+}(\beta) \\
U_{0}^{-}(\beta) & 0
\end{array}\right), \\
& U_{\pi}(\beta, T / 2)=\left(\begin{array}{cc}
U_{\pi}^{+}(\beta) & 0 \\
0 & U_{\pi}^{-}(\beta)
\end{array}\right) .
\end{aligned}
$$

Then, the non-Bloch winding numbers of Yao-Wang formula can be written as following[23, 25]:

$$
W_{\epsilon=0, \pi}=\frac{i}{2 \pi} \int_{C_{\beta}} \operatorname{Tr}\left[\left(U_{\epsilon}^{+}(\beta)\right)^{-1} d U_{\epsilon}^{+}(\beta)\right],
$$

which characterize the numbers of the Floquet zero and $\pi$ modes at the quasienergies of $\epsilon=0, \pi$ as shown in Fig.1(b). Therefore, we construct the non-Hermitian bulk-boundary correspondence that the robust topological nontrivial zero and $\pi$ modes are characterized by the non-Bloch winding numbers $\left(W_{\epsilon=0, \pi}\right)$.

Non-Bloch Band Invariant.- With the help of the Floquet operator $U(\beta, T)$, we can define the non-Bloch band invariant
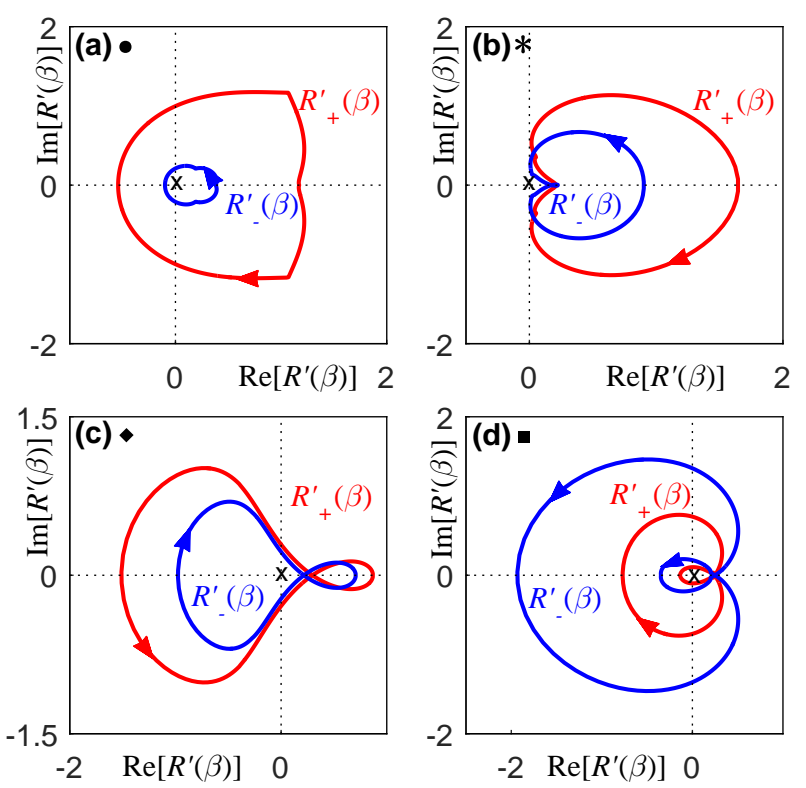

FIG. 2. (a)-(d) show the loops of $R_{+}^{\prime}(\beta)$ (red) and $R_{-}^{\prime}(\beta)$ (blue) on the complex plane along the generalized Brillouin zone $C_{\beta}$ of Fig.1(c)(f), respectively.

for the Floquet bands $[25,91,98,102,110]$. We also can give the non-Bloch band invariant easily and intuitively. For single resonance case, the non-Bloch effective Hamiltonian can be derived from the Floquet Hamiltonian:

$$
H_{\mathrm{eff}}(\beta)=R_{+}^{\prime}(\beta) \sigma_{+}+R_{-}^{\prime}(\beta) \sigma_{-},
$$

with $R_{ \pm}^{\prime}(\beta)=\left[1-\frac{\omega}{2 d_{\beta}} \mp \frac{\lambda\left[R_{+}(\beta)-R_{-}(\beta)\right]}{4 d_{\beta}^{2}}\right] R_{ \pm}(\beta), R_{ \pm}(\beta)=t_{1} \pm \gamma+$ $t_{2} \beta^{\mp}$ and $d_{\beta}=\sqrt{R_{+}(\beta) R_{-}(\beta)}$. The GBZ $C_{\beta}$ can be derived by the eigenvalue equation $E^{2}=R_{+}^{\prime}(\beta) R_{-}^{\prime}(\beta)$ [23, 83], which is consistent with the one derived from the above non-Bloch Floquet Hamiltonian as shown in Fig.1(c)-(f). The GBZs are determined by non-Bloch Floquet Hamiltonian (solid) and nonBloch effective Hamiltonian (dotted) for the four topological different phases. The two curves coincide with each other.

Due to the chiral symmetry of the effective Hamiltonian $\sigma_{z} H_{\mathrm{eff}}(\beta) \sigma_{z}=-H_{\mathrm{eff}}(\beta)$, the generalized ' $\mathrm{Q}$ matrix' $Q(\beta)$ is given by

$$
Q(\beta)=\frac{1}{\sqrt{R_{+}^{\prime}(\beta) R_{-}^{\prime}(\beta)}}\left(\begin{array}{cc}
0 & R_{+}^{\prime}(\beta) \\
R_{-}^{\prime}(\beta) & 0
\end{array}\right) .
$$

Thus, we obtain $q=R_{+}^{\prime}(\beta) / \sqrt{R_{+}^{\prime}(\beta) R_{-}^{\prime}(\beta)}$ and have the YaoWang formula of the non-Bloch band invariant[23, 83] for Floquet bands as:

$$
\begin{aligned}
\mathcal{W} & =\frac{i}{2 \pi} \int_{C_{\beta}} d q q^{-1}(\beta) \\
& =-\frac{1}{4 \pi}\left[\arg R_{+}^{\prime}(\beta)-\arg R_{-}^{\prime}(\beta)\right]_{C_{\beta}} .
\end{aligned}
$$

The non-Bloch band invariant is determined by the changes of the phases of $R_{ \pm}^{\prime}(\beta)$ when $\beta$ goes along GBZ $C_{\beta}$ in the 

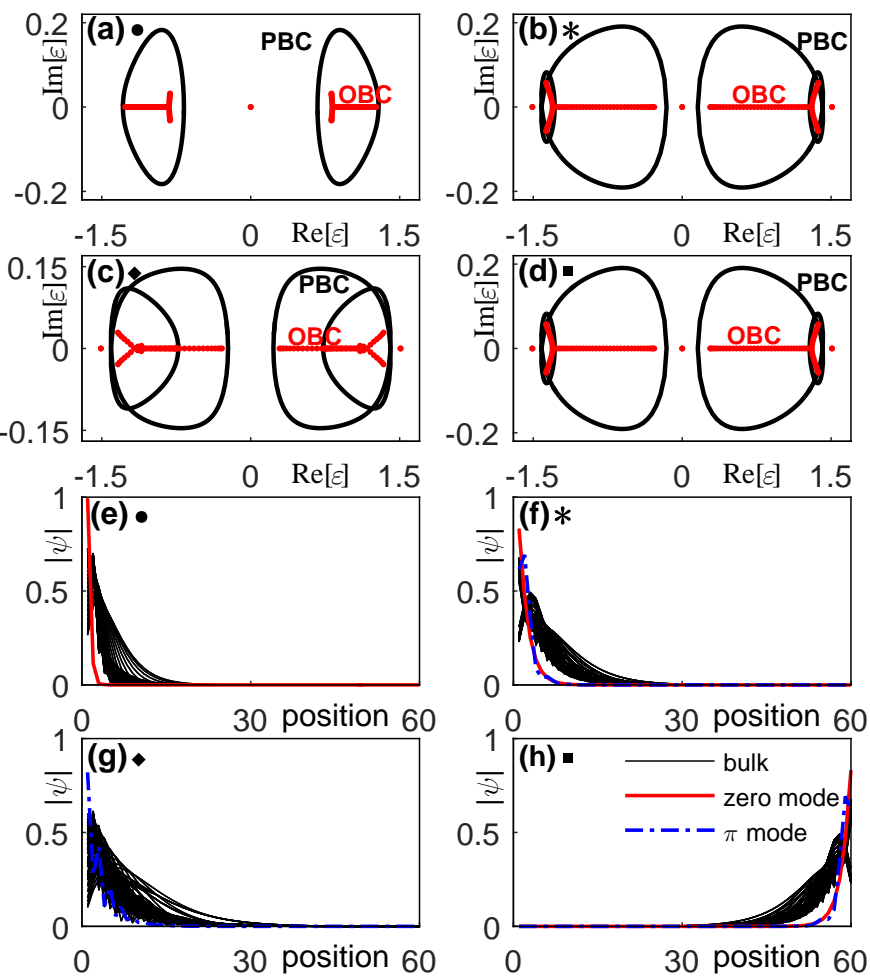

FIG. 3. Non-Hermitian Skin Effect of Floquet System. (a)-(d) The quasienergy spectra under periodic boundary condition (black) and open boundary condition (red) of the four topological different phases in Fig.1(b). The values of the parameters are $\omega=3$ (a) $t_{1}=0.3$, (b) $t_{1}=0.75$, (c) $t_{1}=1.3$ and (d) $t_{1}=-0.75$. (e)-(h) The bulk eigenstates (black) and the zero (red) and $\pi$ (blue) modes of the Floquet Hamiltonian in open boundary condition for (a)-(d), respectively.

counterclockwise way. Fig.2(a)-(d) show the loops of $R_{+}^{\prime}(\beta)$ (red) and $R_{-}^{\prime}(\beta)$ (blue) on the $R^{\prime}$ plane along the GBZ of Fig.1 (c)-(f) in the counterclockwise way. Thus, the non-Bloch band invariants are 1, 0, -1 and 2 for Fig.2(a)-(d), respectively. Fig.2(b) shows that the original point is not encircled by $R_{+}^{\prime}(\beta)$ and $R_{-}^{\prime}(\beta)$, which indicates that the Floquet band is topological trivial $(\mathcal{W}=0)$. While, the phase is topological nontrivial with nonzero non-Bloch winding numbers $\left(W_{0, \pi}=1\right)$ and has topological robust zero and $\pi$ modes. Thus, the nonBloch band invariant only characterizes the topological properties of the Floquet bands and cannot characterizes the robust topological edge modes. This is due to the Floquet operator also lost the information of time-evolution within each period. Fig.2(d) shows that both of the $R_{+}^{\prime}(\beta)$ and $R_{-}^{\prime}(\beta)$ wind the origin point twice but in the opposite direction. The non-Bloch band invariant is 2 with nontrivial non-Bloch winding numbers $W_{0}=1$ and $W_{\pi}=-1$. Certainly, the relation between the non-Bloch winding numbers and the non-Bloch band invariant is $W_{0}-W_{\pi}=\mathcal{W}$ (refer to Supplemental Materials). The non-Bloch winding numbers are more fundamental than the non-Bloch band invariant of the Floquet bands.

Non-Hermitian Skin Effect.- We have shown that the GBZs deviate from the BZ in Fig.1. Undoubtedly, the deviation induces not only the above breakdown of Bloch bulk-

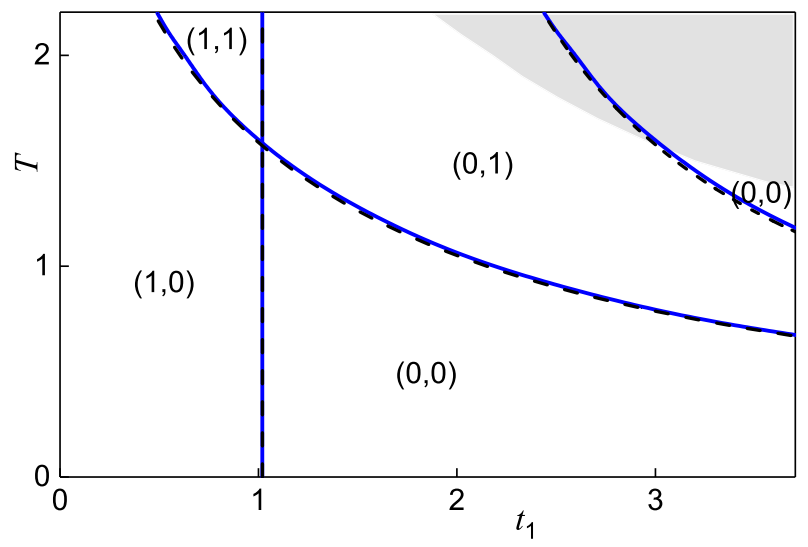

FIG. 4. The Phase Diagram on the $T-t_{1}$ plane: The phase diagram is determined by the non-Bloch band theory (dashed) and the quasienergy of Floquet Hamiltonian (solid) with $t_{2}=1, \gamma=0.2$ and $\lambda=0.5$. The shaded region represents that the non-Bloch topological invariants are not well-defined for the gapless quasienergy. Four topological different phases with non-Bloch winding numbers: $\left(W_{0}, W_{\pi}\right)=(1,0),(0,0),(0,1)$ and $(1,1)$. For $t_{1}<0$, only $W_{\pi}$ changes the sign.

boundary correspondence but the non-Hermitian skin effect, which is another significantly different from Hermitian systems. Fig.3(a)-(d) show the quasienergy spectra of the Floquet Hamiltonian in $\mathrm{OBC}$ (red) and $\mathrm{PBC}$ (black) for the four topological different phases in Fig.1(b). The quasienergy spectra of the Floquet Hamiltonian have essential difference between the $\mathrm{OBC}$ and $\mathrm{PBC}$, which is also induced by the deviation between the GBZ and BZ. Certainly, Floquet Hamiltonian $\mathcal{H}(\beta)$ can undoubtedly give the quasienergy spectra with $\mathrm{OBC}$ and PBC in Fig.3(a)-(d) along the GBZ $C_{\beta}$ and the BZ in Fig.1(c)(f), respectively. This essential difference indicates the emergence of the non-Hermitian skin effect as shown in Fig.3(e)(h). Fig.3(e)-(h) show the eigenstates of the Floquet Hamiltonian in OBC with lattice size $L=60$. All the eigenstates of the non-Hermitian system are localized at the ends, which is called non-Hermitian skin effect. When $|\beta|$ is smaller than 1 , the correspond eigenstate is localized at the left end of the system, Otherwise, the eigenstate is localized at the right end of the system[84]. Accordingly, the eigenstates of the system are localized at the left ends for (e)-(g) and right ends for $(h)$, whether the eigenstates of bulk bands or the two topological different edge modes. This coincides with the non-Hermitian systems without periodic driving[23].

The Phase diagram.- Based on the quasienergies of Floquet Hamiltonian in $\mathrm{OBC}$ and the non-Bloch band theory, we can draw the phase diagram on the $T-t_{1}$ plane with $t_{2}=1$, $\gamma=0.2$ and $\lambda=0.5$ as shown in Fig.4. For weak driving case, the phase boundaries are approximated as $t_{1}= \pm \sqrt{t_{2}^{2}+\gamma^{2}}$ and $\omega=2\left|t_{2} \pm \sqrt{\left|t_{1}^{2}-\gamma^{2}\right|}\right|$, which can be determined by the changing of the non-Bloch band invariant. In the phase diagram, there are four topological different phases: (i) only having zero modes $(1,0)$, (ii) having any edge modes $(0,0)$, (iii) only having $\pi$ modes $(0,1)$ and (iv) having both zero and 
$\pi$ modes $(1,1)$. The Floquet bands are not isolated in the shaded region, where the non-Bloch topological invariants are not well defined.

Conclusion:- We investigated the topological properties of the non-Hermitian Su-Schrieffer-Heeger model with periodic driving which exhibits non-Hermitian skin effect: all the eigenstates are localized at the edges. We found that there are two types of robust edge modes with quasienergies $\epsilon=0, \pi$. The topology of the edge modes cannot be characterized by the winding number defined on the Brillouin zone. Therefore, we established the non-Hermitian bulk-boundary corre- spondence: the robust edge modes can be characterized by the non-Bloch winding numbers defined by the non-Bloch Floquet operators on generalized Brillouin zone. The nonHermitian bulk-boundary correspondence connects the bulk topology and the robust topological edge modes. In our system, we found the robust edge modes can appear when the Floquet bands are topological trivial with zero non-Bloch band invariant.

Acknowledgment:- We thank Zhong Wang for fruitful discussion. This work is supported by NSFC under Grants No.11504143.
[1] M. Z. Hasan and C. L. Kane, "Colloquium: Topological insulators," Rev. Mod. Phys. 82, 3045-3067 (2010).

[2] Xiao-Liang Qi and Shou-Cheng Zhang, "Topological insulators and superconductors," Rev. Mod. Phys. 83, 1057-1110 (2011).

[3] Ching-Kai Chiu, Jeffrey C. Y. Teo, Andreas P. Schnyder, and Shinsei Ryu, "Classification of topological quantum matter with symmetries," Rev. Mod. Phys. 88, 035005 (2016).

[4] A. Bansil, Hsin Lin, and Tanmoy Das, "Colloquium: Topological band theory," Rev. Mod. Phys. 88, 021004 (2016).

[5] C. L. Kane and E. J. Mele, "Quantum spin hall effect in graphene," Phys. Rev. Lett. 95, 226801 (2005).

[6] J. E. Moore and L. Balents, "Topological invariants of time-reversal-invariant band structures," Phys. Rev. B 75, 121306 (2007).

[7] Liang Fu, C. L. Kane, and E. J. Mele, "Topological insulators in three dimensions," Phys. Rev. Lett. 98, 106803 (2007).

[8] B. Andrei Bernevig, Taylor L. Hughes, and Shou-Cheng Zhang, "Quantum spin hall effect and topological phase transition in hgte quantum wells," Science 314, 1757-1761 (2006).

[9] Carl M. Bender and Stefan Boettcher, "Real spectra in non-hermitian hamiltonians having $\mathcal{P T}$ symmetry," Phys. Rev. Lett. 80, 5243-5246 (1998).

[10] Hui Cao and Jan Wiersig, "Dielectric microcavities: Model systems for wave chaos and non-hermitian physics," Rev. Mod. Phys. 87, 61-111 (2015).

[11] M.V. Berry, "Physics of nonhermitian degeneracies," Czech. J. Phys. 54, 1039 (2004).

[12] Youngwoon Choi, Sungsam Kang, Sooin Lim, Wookrae Kim, Jung-Ryul Kim, Jai-Hyung Lee, and Kyungwon An, "Quasieigenstate coalescence in an atom-cavity quantum composite," Phys. Rev. Lett. 104, 153601 (2010).

[13] Sebastian Diehl, Enrique Rico, Mikhail A. Baranov, and Peter Zoller, "Topology by dissipation in atomic quantum wires," Nature Physics 7, 971-977 (2011).

[14] Alois Regensburger, Christoph Bersch, Mohammad-Ali Miri, Georgy Onishchukov, Demetrios N. Christodoulides, and Ulf Peschel, "Parityctime synthetic photonic lattices," Nature 488, 167-171 (2012).

[15] Jan Wiersig, "Enhancing the sensitivity of frequency and energy splitting detection by using exceptional points: Application to microcavity sensors for single-particle detection," Phys. Rev. Lett. 112, 203901 (2014).

[16] Hossein Hodaei, Absar U. Hassan, Steffen Wittek, Hipolito Garcia-Gracia, Ramy El-Ganainy, Demetrios N. Christodoulides, and Mercedeh Khajavikhan, "Enhanced sensitivity at higher-order exceptional points,"
Nature 548, 187-191 (2017).

[17] Ramy El-Ganainy, Konstantinos G. Makris, Mercedeh Khajavikhan, Ziad H. Musslimani, Stefan Rotter, and Demetrios N. Christodoulides, "Non-hermitian physics and pt symmetry," Nature Physics 14, 11 (2018).

[18] Tony E. Lee and Ching-Kit Chan, "Heralded magnetism in non-hermitian atomic systems," Phys. Rev. X 4, 041001 (2014).

[19] Baogang Zhu, Rong Lü, and Shu Chen, "PT symmetry in the non-hermitian su-schrieffer-heeger model with complex boundary potentials," Phys. Rev. A 89, 062102 (2014).

[20] Simon Malzard, Charles Poli, and Henning Schomerus, "Topologically protected defect states in open photonic systems with non-hermitian charge-conjugation and parity-time symmetry," Phys. Rev. Lett. 115, 200402 (2015).

[21] Bo Zhen, Chia Wei Hsu, Yuichi Igarashi, Ling Lu, Ido Kaminer, Adi Pick, Song-Liang Chua, John D. Joannopoulos, and Marin Soljačić, "Spawning rings of exceptional points out of Dirac cones," Nature (London) 525, 354-358 (2015).

[22] Tony E. Lee, "Anomalous edge state in a non-hermitian lattice," Phys. Rev. Lett. 116, 133903 (2016).

[23] Shunyu Yao and Zhong Wang, "Edge states and topological invariants of non-hermitian systems," Phys. Rev. Lett. 121, 086803 (2018).

[24] Shunyu Yao, Fei Song, and Zhong Wang, "Non-hermitian chern bands," Phys. Rev. Lett. 121, 136802 (2018).

[25] Lei Xiao, Tianshu Deng, Kunkun Wang, Gaoyan Zhu, Zhong Wang, Wei Yi, and Peng Xue, "Non-hermitian bulkcboundary correspondence in quantum dynamics," Nature Physics 16, 761 (2020).

[26] Ken-Ichiro Imura and Yositake Takane, "Bloch band theory for non-hermitian bulk-boundary correspondence," arXiv:2004.14772 (2020).

[27] Yong $\mathrm{Xu}$, Sheng-Tao Wang, and L.-M. Duan, "Weyl exceptional rings in a three-dimensional dissipative cold atomic gas," Phys. Rev. Lett. 118, 045701 (2017).

[28] Henri Menke and Moritz M. Hirschmann, "Topological quantum wires with balanced gain and loss," Phys. Rev. B 95, 174506 (2017).

[29] Qi-Bo Zeng, Shu Chen, and Rong Lü, “Anderson localization in the non-hermitian aubry-andré-harper model with physical gain and loss," Phys. Rev. A 95, 062118 (2017).

[30] A. McDonald, T. Pereg-Barnea, and A. A. Clerk, "Phase-dependent chiral transport and effective nonhermitian dynamics in a bosonic kitaev-majorana chain," Phys. Rev. X 8, 041031 (2018).

[31] Tsuneya Yoshida, Robert Peters, and Norio Kawakami, "Non- 
hermitian perspective of the band structure in heavy-fermion systems," Phys. Rev. B 98, 035141 (2018).

[32] Huitao Shen and Liang Fu, "Quantum oscillation from in-gap states and a non-hermitian landau level problem," Phys. Rev. Lett. 121, 026403 (2018).

[33] Johan Carlström and Emil J. Bergholtz, "Exceptional links and twisted fermi ribbons in non-hermitian systems," Phys. Rev. A 98, 042114 (2018).

[34] Ye Xiong, "Why does bulk boundary correspondence fail in some non-hermitian topological models," Journal of Physics Communications 2, 035043 (2018).

[35] Zongping Gong, Yuto Ashida, Kohei Kawabata, Kazuaki Takasan, Sho Higashikawa, and Masahito Ueda, "Topological phases of non-hermitian systems," Phys. Rev. X 8, 031079 (2018).

[36] Huitao Shen, Bo Zhen, and Liang Fu, "Topological band theory for non-hermitian hamiltonians," Phys. Rev. Lett. 120, 146402 (2018).

[37] Yu Chen and Hui Zhai, "Hall conductance of a non-hermitian chern insulator," Phys. Rev. B 98, 245130 (2018).

[38] Flore K. Kunst, Elisabet Edvardsson, Jan Carl Budich, and Emil J. Bergholtz, "Biorthogonal bulkboundary correspondence in non-hermitian systems," Phys. Rev. Lett. 121, 026808 (2018).

[39] Elisabet Edvardsson, Flore K. Kunst, and Emil J. Bergholtz, "Non-hermitian extensions of higher-order topological phases and their biorthogonal bulk-boundary correspondence," Phys. Rev. B 99, 081302 (2019).

[40] Simon Lieu, "Non-hermitian majorana modes protect degenerate steady states," Phys. Rev. B 100, 085110 (2019).

[41] Tsuneya Yoshida and Yasuhiro Hatsugai, "Exceptional rings protected by emergent symmetry for mechanical systems," Phys. Rev. B 100, 054109 (2019).

[42] Tsuneya Yoshida, Robert Peters, Norio Kawakami, and Yasuhiro Hatsugai, "Symmetry-protected exceptional rings in two-dimensional correlated systems with chiral symmetry," Phys. Rev. B 99, 121101 (2019).

[43] Emil J. Bergholtz, Jan Carl Budich, and Flore K. Kunst, "Exceptional topology of non-hermitian systems," arXiv:1912.10048 (2019).

[44] Po-Yao Chang, Jhih-Shih You, Xueda Wen, and Shinsei Ryu, "Entanglement spectrum and entropy in topological nonhermitian systems and non-unitary conformal field theories," arXiv:1909.01346 (2019).

[45] Jonatan Melkær Midtgaard, Zhigang Wu, and Yu Chen, "Constraints on the energy spectrum of non-hermitian models in open environments," arXiv:1905.09603 (2019).

[46] Bastian Höckendorf, Andreas Alvermann, and Holger Fehske, "Non-hermitian boundary state engineering in anomalous floquet topological insulators," Phys. Rev. Lett. 123, 190403 (2019).

[47] Nobuyuki Okuma and Masatoshi Sato, "Topological phase transition driven by infinitesimal instability: Majorana fermions in non-hermitian spintronics," Phys. Rev. Lett. 123, 097701 (2019).

[48] S. Longhi, "Topological phase transition in non-hermitian quasicrystals," Phys. Rev. Lett. 122, 237601 (2019).

[49] Zhesen Yang and Jiangping Hu, "Non-hermitian hopf-link exceptional line semimetals,” Phys. Rev. B 99, 081102 (2019).

[50] Kohei Kawabata, Sho Higashikawa, Zongping Gong, Yuto Ashida, and Masahito Ueda, "Topological unification of time-reversal and particle-hole symmetries in non-hermitian physics," Nature Communications 10, 297 (2019).

[51] Bastian Höckendorf, Andreas Alvermann, and
Holger Fehske, "Topological origin of quantized transport in non-hermitian floquet chains," Phys. Rev. Research 2, 023235 (2020).

[52] Xiao-Ran Wang, Cui-Xian Guo, and Su-Peng Kou, "Defective edge states and number-anomalous bulk-boundary correspondence in non-hermitian topological systems," Phys. Rev. B 101, 121116 (2020).

[53] Cui-Xian Guo, Xiao-Ran Wang, Can Wang, and Su-Peng Kou, "Non-hermitian dynamic strings and anomalous topological degeneracy on a non-hermitian toric-code model with parity-time symmetry," Phys. Rev. B 101, 144439 (2020).

[54] Zhesen Yang, Ching-Kai Chiu, Chen Fang, and Jiangping $\mathrm{Hu}$, "Jones polynomial and knot transitions in hermitian and non-hermitian topological semimetals," Phys. Rev. Lett. 124, 186402 (2020).

[55] T. Helbig, T. Hofmann, S. Imhof, M. Abdelghany, T. Kiessling, L. W. Molenkamp, C. H. Lee, A. Szameit, M. Greiter, and R. Thomale, "Generalized bulkcboundary correspondence in non-hermitian topolectrical circuits," Nature Physics 16, 747 (2020).

[56] Lei Pan, Xin Chen, Yu Chen, and Hui Zhai, "Non-hermitian linear response theory," Nature Physics 16, 767 (2020).

[57] Tsuneya Yoshida, Tomonari Mizoguchi, and Yasuhiro Hatsugai, "Mirror skin effect and its electric circuit simulation," Phys. Rev. Research 2, 022062 (2020).

[58] Dan S. Borgnia, Alex Jura Kruchkov, and Robert-Jan Slager, "Non-hermitian boundary modes and topology," Phys. Rev. Lett. 124, 056802 (2020).

[59] Qi-Bo Zeng, Yan-Bin Yang, and Yong Xu, "Topological phases in non-hermitian aubry-andré-harper models," Phys. Rev. B 101, 020201 (2020).

[60] P. O. Sukhachov and A. V. Balatsky, "Non-hermitian impurities in dirac systems," Phys. Rev. Research 2, 013325 (2020).

[61] Zhen-Qian Yang, Zeng-Kai Shao, Hua-Zhou Chen, Xin-Rui Mao, and Ren-Min Ma, "Spin-momentumlocked edge mode for topological vortex lasing," Phys. Rev. Lett. 125, 013903 (2020).

[62] Haoran Xue, Qiang Wang, Baile Zhang, and Y. D. Chong, "Non-hermitian dirac cones," Phys. Rev. Lett. 124, 236403 (2020).

[63] Kang Yang, Siddhardh C. Morampudi, and Emil J. Bergholtz, "Exceptional spin liquids," arXiv:2007.04329 (2020).

[64] V. M. Martinez Alvarez, J. E. Barrios Vargas, and L. E. F. Foa Torres, "Non-hermitian robust edge states in one dimension: Anomalous localization and eigenspace condensation at exceptional points," Phys. Rev. B 97, 121401 (2018).

[65] Stefano Longhi, "Probing non-hermitian skin effect and non-bloch phase transitions," Phys. Rev. Research 1, 023013 (2019).

[66] Hui Jiang, Li-Jun Lang, Chao Yang, Shi-Liang Zhu, and Shu Chen, "Interplay of non-hermitian skin effects and anderson localization in nonreciprocal quasiperiodic lattices," Phys. Rev. B 100, 054301 (2019).

[67] Ching Hua Lee, Linhu Li, and Jiangbin Gong, "Hybrid higher-order skin-topological modes in nonreciprocal systems," Phys. Rev. Lett. 123, 016805 (2019).

[68] Huaiqiang Wang, Jiawei Ruan, and Haijun Zhang, "Nonhermitian nodal-line semimetals with an anomalous bulkboundary correspondence," Phys. Rev. B 99, 075130 (2019).

[69] Tao Liu, Yu-Ran Zhang, Qing Ai, Zongping Gong, Kohei Kawabata, Masahito Ueda, and Franco Nori, "Second-order topological phases in non-hermitian systems," Phys. Rev. Lett. 122, 076801 (2019).

[70] Fei Song, Shunyu Yao, and Zhong Wang, "Non-hermitian 
skin effect and chiral damping in open quantum systems," Phys. Rev. Lett. 123, 170401 (2019).

[71] Xi-Wang Luo and Chuanwei Zhang, "Higher-order topological corner states induced by gain and loss," Phys. Rev. Lett. 123, 073601 (2019).

[72] Ching Hua Lee, Linhu Li, Ronny Thomale, and Jiangbin Gong, "Unraveling non-hermitian pumping: emergent spectral singularities and anomalous responses," arXiv:1912.06974 (2019).

[73] Linhu Li, Ching Hua Lee, Sen Mu, and Jiangbin Gong, "Critical non-hermitian skin effect," arXiv:2003.03039 (2020).

[74] Ching Hua Lee and Stefano Longhi, "Ultrafast and anharmonic rabi oscillations between non-bloch-bands," arXiv:2003.10763 (2020).

[75] Taiki Haga, Masaya Nakagawa, Ryusuke Hamazaki, and Masahito Ueda, "Liouvillian skin effect: Slowing down of relaxation processes without gap closing," arXiv:2005.00824 (2020).

[76] Yifei Yi and Zhesen Yang, "Non-hermitian skin modes induced by on-site dissipations and chiral tunneling effect," arXiv:2003.02219 (2020).

[77] Nobuyuki Okuma, Kohei Kawabata, Ken Shiozaki, and Masatoshi Sato, "Topological origin of non-hermitian skin effects," Phys. Rev. Lett. 124, 086801 (2020).

[78] C. Yuce, "Non-hermitian anomalous skin effect," Physics Letters A 384, 126094 (2020).

[79] Qi-Bo Zeng and Yong $\mathrm{Xu}$, "Winding numbers and generalized mobility edges in non-hermitian systems," Phys. Rev. Research 2, 033052 (2020).

[80] Chun-Hui Liu, Kai Zhang, Zhesen Yang, and Shu Chen, "Helical damping and anomalous critical non-hermitian skin effect," arXiv:2005.02617 (2020).

[81] Linhu Li, Ching Hua Lee, and Jiangbin Gong, "Topological switch for non-hermitian skin effect in cold-atom systems with loss," Phys. Rev. Lett. 124, 250402 (2020).

[82] Tian-Shu Deng and Wei Yi, "Non-bloch topological invariants in a non-hermitian domain wall system," Phys. Rev. B 100, 035102 (2019).

[83] Kazuki Yokomizo and Shuichi Murakami, "Nonbloch band theory of non-hermitian systems," Phys. Rev. Lett. 123, 066404 (2019).

[84] Fei Song, Shunyu Yao, and Zhong Wang, "Nonhermitian topological invariants in real space," Phys. Rev. Lett. 123, 246801 (2019).

[85] Kai Zhang, Zhesen Yang, and Chen Fang, "Correspondence between winding numbers and skin modes in non-hermitian systems," arXiv:1910.01131 (2019).

[86] S. Longhi, "Non-bloch-band collapse and chiral zener tunneling," Phys. Rev. Lett. 124, 066602 (2020).

[87] Ching Hua Lee and Ronny Thomale, "Anatomy of skin modes and topology in non-hermitian systems," Phys. Rev. B 99, 201103 (2019).

[88] Kohei Kawabata, Ken Shiozaki, Masahito Ueda, and Masatoshi Sato, "Symmetry and topology in non-hermitian physics," Phys. Rev. X 9, 041015 (2019).

[89] Wen-Tan Xue, Ming-Rui Li, Yu-Min Hu, Fei Song, and Zhong Wang, "Non-hermitian band theory of directional amplification,” arXiv:2004.09529 (2020).

[90] Zhesen Yang, Kai Zhang, Chen Fang, and Jiangping $\mathrm{Hu}$, "Auxiliary generalized brillouin zone method in nonhermitian band theory," arXiv:1912.05499 (2019).

[91] Hong Wu and Jun-Hong An, "Floquet topological phases of non-hermitian systems," Phys. Rev. B 102, 041119 (2020).

[92] Bastian Höckendorf, Andreas Alvermann, and Hol- ger Fehske, "Non-hermitian boundary state engineering in anomalous floquet topological insulators," Phys. Rev. Lett. 123, 190403 (2019).

[93] Tobias Hofmann, Tobias Helbig, Frank Schindler, Nora Salgo, Marta Brzezińska, Martin Greiter, Tobias Kiessling, David Wolf, Achim Vollhardt, Anton Kabaši, Ching Hua Lee, Ante Bilušić, Ronny Thomale, and Titus Neupert, "Reciprocal skin effect and its realization in a topolectrical circuit," Phys. Rev. Research 2, 023265 (2020).

[94] Ananya Ghatak, Martin Brandenbourger, Jasper van Wezel, and Corentin Coulais, "Observation of nonhermitian topology and its bulk-edge correspondence," arXiv:1907.11619 (2019).

[95] Lih-King Lim, C. Morais Smith, and Andreas Hemmerich, "Staggered-vortex superfluid of ultracold bosons in an optical lattice," Phys. Rev. Lett. 100, 130402 (2008).

[96] P. Soltan-Panahi, J. Struck, P. Hauke, A. Bick, W. Plenkers, G. Meineke, C. Becker, P. Windpassinger, M. Lewenstein, and K. Sengstock, "Multi-component quantum gases in spin-dependent hexagonal lattices," Nature Physics 7, 434-440 (2011).

[97] Netanel H. Lindner, Gil Refael, and Victor Galitski, "Floquet topological insulator in semiconductor quantum wells," Nature Physics 7, 490-495 (2011).

[98] Mark S. Rudner, Netanel H. Lindner, Erez Berg, and Michael Levin, "Anomalous edge states and the bulk-edge correspondence for periodically driven two-dimensional systems," Phys. Rev. X 3, 031005 (2013).

[99] Mikael C. Rechtsman, Julia M. Zeuner, Yonatan Plotnik, Yaakov Lumer, Daniel Podolsky, Felix Dreisow, Stefan Nolte, Mordechai Segev, and Alexander Szameit, "Photonic floquet topological insulators," Nature 496, 196-200 (2013).

[100] Michel Fruchart, "Complex classes of periodically driven topological lattice systems," Phys. Rev. B 93, 115429 (2016).

[101] André Eckardt, "Colloquium: Atomic quantum gases in periodically driven optical lattices," Rev. Mod. Phys. 89, 011004 (2017).

[102] Shunyu Yao, Zhongbo Yan, and Zhong Wang, "Topological invariants of floquet systems: General formulation, special properties, and floquet topological defects," Phys. Rev. B 96, 195303 (2017).

[103] Xiaosen Yang, Beibing Huang, and Zhengling Wang, "Floquet topological superfluid and majorana zero modes in two-dimensional periodically driven fermi systems," Scientific Reports 8, 2243 (2018).

[104] Longwen Zhou and Jiangbin Gong, "Non-hermitian floquet topological phases with arbitrarily many real-quasienergy edge states," Phys. Rev. B 98, 205417 (2018).

[105] Li He, Zachariah Addison, Jicheng Jin, Eugene J. Mele, Steven G. Johnson, and Bo Zhen, "Floquet chern insulators of light," Nature Communications 10, 4194 (2019).

[106] Mengyao Li, Xiang Ni, Matthew Weiner, Andrea Alù, and Alexander B. Khanikaev, "Topological phases and nonreciprocal edge states in non-hermitian floquet insulators," Phys. Rev. B 100, 045423 (2019).

[107] Longwen Zhou, "Non-hermitian floquet topological superconductors with multiple majorana edge modes," Phys. Rev. B 101, 014306 (2020).

[108] Xizheng Zhang and Jiangbin Gong, "Non-hermitian floquet topological phases: Exceptional points, coalescent edge modes, and the skin effect," Phys. Rev. B 101, 045415 (2020).

[109] Peng He and Ze-Hao Huang, "Floquet-engineering and simulating exceptional rings with a quantum spin system," arXiv:2005.02703 (2020). 
[110] Tianyu Li, Yong-Sheng Zhang, and Wei Yi, "A twodimensional quantum walk with non-hermitian skin effects," arXiv:2005.09474 (2020). 


\section{Supplemental Materials}

Symmetry:- The chiral symmetry of the non-Bloch Hamiltonian:

$$
\sigma_{z} H(\beta, t) \sigma_{z}=-H(\beta,-t) .
$$

The non-Bloch evolution operator:

$$
\begin{aligned}
& \sigma_{z} U(\beta, t) \sigma_{z} \\
= & \sigma_{z} \mathcal{T}\left[e^{-i \int_{0}^{t} d t^{\prime} H\left(\beta, t^{\prime}\right)}\right] \sigma_{z} \\
= & \sigma_{z}\left[e^{-i \Delta t H(\beta, t)} \cdots e^{-i \Delta t H(\beta, \Delta t)}\right] \sigma_{z} \\
= & \sigma_{z}[1-i \Delta t H(\beta, t)] \sigma_{z} \cdots \sigma_{z}^{-1}[1-i \Delta t H(\beta, \Delta t)] \sigma_{z} \\
= & {[1+i \Delta t H(\beta,-t)] \cdots[1+i \Delta t H(\beta,-\Delta t)] } \\
= & \{[1-i \Delta t H(\beta,-\Delta t)] \cdots[1-i \Delta t H(\beta,-t)]\}^{-1} \\
= & U^{-1}(\beta, t)=U(\beta,-t)
\end{aligned}
$$

The effective non-Bloch Hamiltonian:

$$
\begin{aligned}
& \sigma_{z} H_{\mathrm{eff}}^{\epsilon}(\beta) \sigma_{z} \\
= & \frac{i}{T} \ln _{-\epsilon}\left[\sigma_{z} U(\beta, T) \sigma_{z}\right] \\
= & \frac{i}{T} \ln _{-\epsilon}\left[U^{-1}(\beta, T)\right] \\
= & \frac{i}{T} \sum_{n} \ln _{-\epsilon}\left(\lambda_{n}^{-1}\right)\left|\psi_{n, R}(\beta)\right\rangle\left\langle\psi_{n, L}(\beta)\right| \\
= & \frac{i}{T} \sum_{n}\left[-\ln _{-\epsilon}\left(\lambda_{n}\right)-2 \pi i\right]\left|\psi_{n, R}(\beta)\right\rangle\left\langle\psi_{n, L}(\beta)\right| \\
= & -H_{\mathrm{eff}}^{-\epsilon}(\beta)+\omega
\end{aligned}
$$

The non-Bloch periodized evolution operator:

$$
\begin{aligned}
& \sigma_{z} U_{\epsilon}(\beta, t) \sigma_{z} \\
= & \sigma_{z} U(\beta, t) \sigma_{z} \sigma_{z} \exp \left[i H_{\mathrm{eff}}^{\epsilon}(\beta) t\right] \sigma_{z} \\
= & U(\beta,-t) \exp \left[i \sigma_{z} H_{\mathrm{eff}}^{\epsilon}(\beta) \sigma_{z} t\right] \\
= & U(\beta,-t) \exp \left[-i H_{\mathrm{eff}}^{-\epsilon}(\beta) t+i \omega t\right] \\
= & U_{-\epsilon}(\beta,-t) \exp [i \omega t] .
\end{aligned}
$$

For $\epsilon=0, \pi$, we have

$$
\begin{aligned}
& \sigma_{z} U_{0}\left(\beta, \frac{T}{2}\right) \sigma_{z}=-U_{0}\left(\beta, \frac{T}{2}\right), \\
& \sigma_{z} U_{\pi}\left(\beta, \frac{T}{2}\right) \sigma_{z}=+U_{\pi}\left(\beta, \frac{T}{2}\right) .
\end{aligned}
$$

Then, $U_{0, \pi}$ takes the following forms:

$$
\begin{aligned}
& U_{0}(\beta, T / 2)=\left(\begin{array}{cc}
0 & U_{0}^{+}(\beta) \\
U_{0}^{-}(\beta) & 0
\end{array}\right), \\
& U_{\pi}(\beta, T / 2)=\left(\begin{array}{cc}
U_{\pi}^{+}(\beta) & 0 \\
0 & U_{\pi}^{-}(\beta)
\end{array}\right) .
\end{aligned}
$$

Then,

$$
U_{\pi}^{-1}\left(\frac{T}{2}\right) U_{0}\left(\frac{T}{2}\right)=\left(\begin{array}{cc}
0 & {\left[U_{\pi}^{+}\right]^{-1} U_{0}^{+}} \\
{\left[U_{\pi}^{-}\right]^{-1} U_{\pi}^{-}} & 0
\end{array}\right) .
$$

Relationship between the non-Bloch winding numbers and non-Bloch Band invariant:- We prove the relation between the non-Bloch winding numbers and the the non-Bloch band invariant $\mathcal{W}\left(P_{0, \pi}\right)=W\left[U_{0}\left(\beta, \frac{T}{2}\right)\right]-W\left[U_{\pi}\left(\beta, \frac{T}{2}\right)\right]$ in the main text. The difference between the two non-Bloch effective Hamiltonian is:

$$
\begin{aligned}
& H_{\mathrm{eff}}^{\pi}(\beta)-H_{\mathrm{eff}}^{0}(\beta) \\
= & \frac{i}{T} \ln _{-\pi} U(\beta, T)-\frac{i}{T} \ln _{0} U(\beta, T) \\
= & \frac{i}{T} \sum_{n}\left[\ln _{-\pi}\left(\lambda_{n}\right)-\ln _{0}\left(\lambda_{n}\right)\right]\left|\psi_{n, R}(\beta)\right\rangle\left\langle\psi_{n, L}(\beta)\right| \\
= & \left.\frac{i}{T} \sum_{-\pi<\epsilon_{n}<0}+\sum_{0<\epsilon_{n}<\pi}\right)\left[\ln _{-\pi}\left(e^{-i \epsilon_{n}}\right)-\ln _{0}\left(e^{-i \epsilon_{n}}\right)\right]\left|\psi_{n, R}(\beta)\right\rangle\left\langle\psi_{n, L}(\beta)\right| \\
= & \frac{i}{T} \sum_{0<\epsilon_{n}<\pi}\left[-i \epsilon_{n}-2 \pi i+i \epsilon_{n}\right]\left|\psi_{n, R}(\beta)\right\rangle\left\langle\psi_{n, L}(\beta)\right| \\
= & \frac{2 \pi}{T} \sum_{0<\epsilon_{n}<\pi}\left|\psi_{n, R}(\beta)\right\rangle\left\langle\psi_{n, L}(\beta)\right| \\
= & \omega P_{0, \pi}(\beta) .
\end{aligned}
$$

The non-Bloch periodized evolution operator at half period:

$$
\begin{aligned}
& U_{\pi}^{-1}\left(\beta, \frac{T}{2}\right) U_{0}\left(\beta, \frac{T}{2}\right) \\
= & \exp \left[-i H_{\mathrm{eff}}^{\pi}(\beta) \frac{T}{2}\right] U\left(\beta,-\frac{T}{2}\right) U\left(\beta, \frac{T}{2}\right) \exp \left[i H_{\mathrm{eff}}^{0}(\beta) \frac{T}{2}\right] \\
= & \exp \left(-i \pi P_{0, \pi}\right) \\
= & 1-2 P_{0, \pi} \\
= & Q(\beta) .
\end{aligned}
$$

For the winding number, there is additive property:

$W\left[U_{\pi}^{-1}\left(\beta, \frac{T}{2}\right) U_{0}\left(\beta, \frac{T}{2}\right)\right]=W\left[U_{0}\left(\beta, \frac{T}{2}\right)\right]-W\left[U_{\pi}\left(\beta, \frac{T}{2}\right)\right]$.

Therefore, the relationship between the non-Bloch winding numbers and the non-Bloch band invariant can be written as following:

$$
W\left[U_{0}\left(\beta, \frac{T}{2}\right)\right]-W\left[U_{\pi}\left(\beta, \frac{T}{2}\right)\right]=W .
$$

Topological Phase Boundaries Based on the Effective Hamiltonian:- The non-Bloch effective Hamiltonian is defined by the non-Bloch Floquet operator:

$$
\begin{aligned}
H_{\mathrm{eff}}(\beta) & =\frac{i}{T} \ln U(\beta, T), \\
& =H_{0}(\beta)+\sum_{n \neq 0} \frac{\left[H_{0}, H_{n}\right]}{n \omega}+\sum_{n>0} \frac{\left[H_{n}, H_{-n}\right]}{n \omega} .
\end{aligned}
$$

Here, $H_{0}(\beta)=R_{+}(\beta) \sigma_{+}+R_{-}(\beta) \sigma_{-}$and $H_{ \pm 1}(\beta)=\frac{\lambda}{2} \sigma_{x}$ with $R_{ \pm}(\beta)=t_{1} \pm \gamma+t_{2} \beta^{\mp}$.

For high frequency case $(\omega>\Lambda, \Lambda$ is the bandwidth of $H_{0}$ ), the effective Hamiltonian is not effected by the periodic driving and can be written as $H_{\text {eff }}(\beta)=R_{+}^{\prime}(\beta) \sigma_{+}+R_{-}^{\prime}(\beta) \sigma_{-}$ with $R_{ \pm}^{\prime}(\beta)=R_{ \pm}(\beta)$. The generalized Brilouin zone is a circle $C_{\beta}=\sqrt{\frac{t_{1}-\gamma}{t_{1}+\gamma} \mid} e^{i \theta}$ with $\theta=[0,2 \pi)$. 


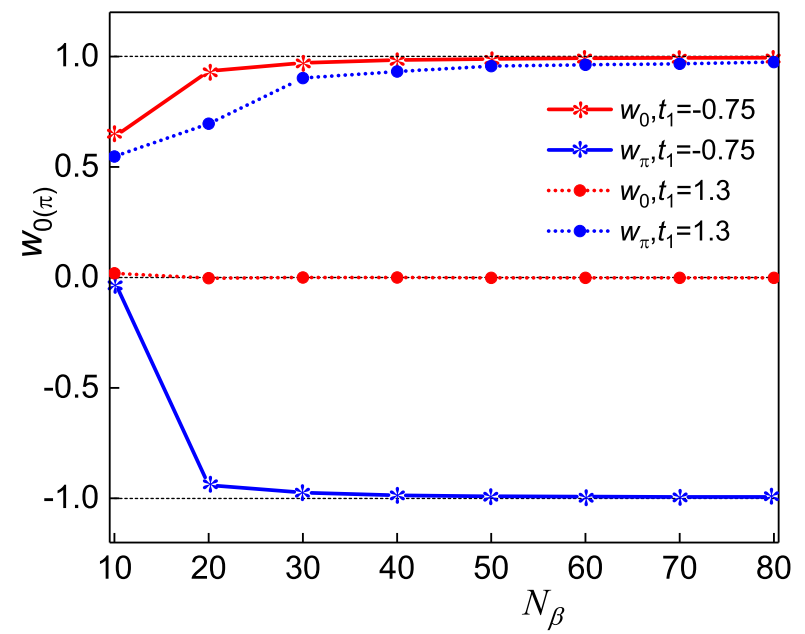

FIG. 5. Non-Bloch winding numbers $W_{0}$ and $W_{\pi}$ as functions of the GBZ size $\left(N_{\beta}\right)$ for $t_{1}=-0.75$ (solid) and $t_{1}=1.3$ (dotted), with $t_{2}=1$, $\gamma=0.2, \lambda=0.5, \omega=3$. Increasing the size, the non-Bloch winding numbers quickly approach into an integer.

For low frequency case $(\omega<\Lambda)$, the drive creates a single resonance between the two bulk bands of $H_{0}$. Therefore, the non-Bloch effective Hamiltonian $H_{\mathrm{eff}}(\beta)$ is defined by the rotated Floquet operator $U(\beta, T)=\mathcal{T} \exp \left[-i \int_{0}^{T} d t H_{\text {rot }}(\beta, t)\right]$. The rotated Hamiltonian $H_{\text {rot }}(\beta, t)$ can be deduced by applying a rotation $\hat{O}(\beta, t)=\exp \left[-i \hat{\mathbf{d}}_{\beta} \cdot \sigma \omega t / 2\right]$ to the non-Bloch
Hamiltonian $H(\beta, t)$ with $\hat{\mathbf{d}}_{\beta}=\mathbf{d}_{\beta} / d_{\beta}$ with $d_{\beta}=\sqrt{d_{x}^{2}+d_{y}^{2}}$. Then, the non-Bloch effective Hamiltonian $H_{\text {eff }}(\beta)$ can be rewritten as following:

$$
H_{\mathrm{eff}}(\beta)=R_{+}^{\prime}(\beta) \sigma_{+}+R_{-}^{\prime}(\beta) \sigma_{-},
$$

with

$$
R_{ \pm}^{\prime}(\beta)=\left[1-\frac{\omega}{2 d_{\beta}} \mp \frac{\lambda\left[R_{+}(\beta)-R_{-}(\beta)\right]}{4 d_{\beta}^{2}}\right] R_{ \pm}(\beta) .
$$

For weak driving, the formula of $R^{\prime}$ can be rewritten as

$$
R_{ \pm}^{\prime}(\beta) \simeq\left[1-\frac{\omega}{2 d_{\beta}}\right] R_{ \pm}(\beta) .
$$

Then, the generalized Brilouin zone can be determined and written as

$$
C_{\beta}=\sqrt{\left|\frac{t_{1}-\gamma}{t_{1}+\gamma}\right|} e^{i \theta}
$$

with $\theta=[0,2 \pi)$.

The gap closing equation can be derived directly: $R_{+}(\beta) R_{-}(\beta)=0$ and $\omega=2 d_{\beta}$.

Thus, the topological phase boundaries can be written as following:

$$
\begin{aligned}
& t_{1}= \pm \sqrt{t_{2}^{2}+\gamma^{2}} \\
& \omega=2 \mid t_{2} \pm \sqrt{\left|t_{1}^{2}-\gamma^{2}\right|} .
\end{aligned}
$$

